\title{
Paradigmas de evaluación: del tradicional al socioformativo
}

\section{Evaluation Paradigms: from the Traditional to the Socio-formative}

DOl: https://doi.org/10.32870/dse.v0i21.646

\author{
Ma. de la Luz Berlanga Ramírez* \\ Luis Gibran Juárez-Hernández**
}

\begin{abstract}
Resumen
La evaluación ha sido objeto de diversos estudios en la educación, los cuales han propiciado su evolución gracias a las aportaciones de cada modelo para ir perfeccionando su ejercicio. En el presente ensayo se abordan los siguientes paradigmas de evaluación: tradicional, democrático, basado en criterios, comprensivo o interpretativo, para la toma de decisiones, negociación, calidad o institucional, alternativa, de ejecución, auténtica, progresiva, por competencias y el más reciente, el socioformativo. El objetivo del trabajo es examinar las contribuciones de los paradigmas de evaluación en el proceso de enseñanza - aprendizaje. Se encuentra que algunos de los modelos de evaluación se contraponen, pero otros también se complementan, facilitando la mejora de su aplicación y, por ende, su evolución. La evaluación socioformativa ha tenido contribuciones sustanciales para la evaluación por competencias, además, responde a las necesidades de la sociedad del conocimiento.
\end{abstract}

Palabras clave: evaluación socioformativa - evolución de la evaluación - paradigmas de evaluación - retroalimentación.

\begin{abstract}
Evaluation has been the subject of various studies in education, which have led to its evolution thanks to the contributions of each evaluation model to improve its implementation. This essay addresses thirteen evaluation paradigms: Traditional, Democratic, Criterion-Based, Comprehensive or Interpretive, DecisionMaking Oriented, Negotiation, Quality or Institutional, Alternative, Execution, Authentic, Progressive, Competency-Based, and Socio-formative, the most recent. The aim of this paper is to examine the contributions of the evaluation paradigms to the teaching - learning process. It has been found that some of the evaluation models are opposed but others also complement each other, facilitating the improvement of the
\end{abstract}

\footnotetext{
* Máster en Educación basada en competencias por la Universidad del Valle de México. Doctorando del Centro Universitario CIFE. Profesorainvestigadora de la Escuela Normal Superior del Estado de Coahuila, México. Líneas de investigación: educación y evaluación normalista. maryluzberlanga@cife.edu.mx

**Doctor en Ciencias Biológicas y de la Salud. Profesor-investigador del Centro Universitario CIFE en Cuernavaca, Morelos. México. Líneas de investigación: instrumentos de evaluación y estadística. luisgibran@cife.edu.mx
} 
application of evaluation and, therefore, its evolution. Socio-formative evaluation has made substantial contributions to evaluation by competencies, and responds to needs of the information society.

Keywords: socioformative evaluation - evolution of evaluation - evaluation paradigms - feedback.

\section{Introducción}

Cada vez más la sociedad toma conciencia de la importancia de la evaluación. Desde cualquier ámbito, sea empresarial, organizacional, educativo e incluso, hasta en lo familiar. Asimismo, se afirma que la evaluación involucra lo que se evalúa, a los sujetos o actores y el contexto en el que se genera. En el proceso de aprendizaje, la evaluación se ha estudiado desde varias perspectivas con fines y objetivos diversos. Actualmente existen varios paradigmas de evaluación que proporcionan recursos y plantean metodologías con mayor precisión y claridad, como es el caso del enfoque socioformativo, modelo de evaluación que logra satisfacer las exigencias propias de la sociedad del conocimiento puesto que promueve - entre otros aspectos - el trabajo colaborativo para la resolución de problemas con apoyo de las Tecnologías de la Información y la Comunicación (TIC), Tecnologías del Empoderamiento y la Participación (TEP), Tecnologías del Aprendizaje y el Conocimiento (TAC) y, recientemente, Tecnologías para la Socioformación (TS).

Se han realizado diversas investigaciones acerca de la historia de los paradigmas de evaluación (Alcaraz, 2015; Mejía, 2012; Muñoz, 2007; Rivera de Mendoza y Piñero, 2006; Mora, 2004); sin embargo, es relevante revisar algunas de ellas con el propósito de comprender su evolución tanto conceptual como metodológica. Partiendo del propósito establecido, el presente artículo analiza tal evolución y establece las siguientes metas: 1) Examinar los modelos de evaluación que han influido en el proceso de aprendizaje; 2) Comparar las aportaciones de los paradigmas de evaluación en relación con las necesidades de la sociedad del conocimiento; y 3) Vincular los preceptos pedagógicos de los paradigmas de evaluación con el más actual, el enfoque socioformativo.

\section{Paradigmas de evaluación: del tradicional al socioformativo}

El modelo de evaluación tradicional tiene sus cimientos en el positivismo, durante la época moderna europea. La realidad se examinaba en las ciencias exactas con procedimientos observables, medibles y verificables. Desde estos criterios, el proceso educativo era objeto de observación y medición, de aquí que la evaluación se inclinaba a ser de carácter cuantitativo. En el paradigma tradicional de evaluación se emplea un solo instrumento: el examen o test. Se confeccionan los primeros instrumentos para medir, por ejemplo, las pruebas para evaluar la lectura y escritura: escalas de ortografía de Buckingan, de escritura de Ayres y Freeman y, de redacción de Hillegas, entre otras.

Diólo pos sobre Educación año 11 | número 21 | julio-diciembre 2020 | ISSN 2007-2171 
El sistema de evaluación se caracterizó por ser mecánico, con parámetros estandarizados y masivos, pero no respondían a la información requerida para mejorar el proceso de aprendizaje ni el rendimiento académico del alumno. No se podían evaluar procesos cognitivos superiores porque resultaban ser difícilmente objetos de observación y medición. La memorización era el único proceso cognitivo para evaluarse; fue imposible propiciar el desarrollo del pensamiento analítico, crítico, reflexivo y creativo en el alumno.

En las prácticas educativas, la evaluación tradicional constituye el objetivo primordial de la educación; se aprendía para la evaluación. Es fundamental indicar que la evaluación tradicional no reconoce diversidad cultural, de capacidades, estilos de aprendizaje, entre otros aspectos de los alumnos; es una evaluación homogénea. No otorga espacio a la retroalimentación, ni oportunidades de mejora del aprendizaje; por lo tanto, tampoco se propicia la evaluación procesual ni se considera la evaluación diagnóstica. Tobón (2017) señala que el modelo de evaluación tradicional se caracteriza por varios aspectos, entre los que destacan: que la evaluación es la finalidad primordial; el único que la ejecuta es el maestro y, además, determina arbitrariamente los parámetros sin considerar criterios académicos y profesionales; la información obtenida de la evaluación se otorga mediante notas cuantitativas sin argumentación; se centra más en los errores que en los aciertos; los errores se castigan y no se asumen como oportunidades de aprendizaje; no se brinda la oportunidad de mejoras porque los resultados de las pruebas son definitivos y, por ende, no se proporcionan herramientas para lograrlas.

Con base en la literatura existente, se advierten dos épocas que anteceden a la primera generación establecida por Muñoz (2007): la pretyleriana (2000 a.C. a 1930) y el periodo tyleriano (de 1930 a 1957). En la primera etapa, cuando se genera la medida, el evaluador es comprendido como un proveedor de instrumentos de medición de las destrezas y procesos cognitivos de los alumnos. Las evaluaciones estandarizadas adquieren un auge importante en la educación durante esa época, aunque no suministraba información acerca del currículo. Al respecto, Alcaraz (2015) y Gronlund (1985) coinciden en que esa visión todavía se sigue permeando en la evaluación educativa. La segunda etapa, la tyleriana o descriptiva, se distingue porque cambia la perspectiva acerca de la evaluación tomando un lugar sobre la medición. Según Tyler (1974), la evaluación es la información que facilita la comparación entre los resultados y los objetivos proyectados. No obstante, Escudero (2003), y Stufflebeam y Shinkfield (2005) sostienen que desde esa visión la evaluación no proveía información para mejorar el proceso enseñanza - aprendizaje.

De acuerdo con la clasificación de Muñoz (2007), la primera generación surge en la década de 1960; en este periodo se buscaba medir el dominio de los contenidos a través de test de memoria. Durante la segunda generación, con un enfoque más cualitativo, nace la evaluación de los programas y se presentan dos paradigmas: el de la evaluación basada en criterios y otra comprensiva o interpretativa (Sakate, 2004); la primera evalúa variables descriptivas, y la segun- 
da, el conocimiento experiencial. En la evaluación de la segunda generación se incluyen otros factores: los programas, los currículos, las estrategias didácticas, los materiales, la organización, entre otros. La ventaja de este tipo de evaluación es que proporciona información acerca de las fortalezas y debilidades de un todo integrado, pero se produce una vinculación superlativa entre el objetivo y la evaluación. El evaluador es un descriptor y mediador que ayuda a todos los actores a seleccionar, organizar y formular todo lo operativo en la institución educativa.

La evaluación para la toma de decisiones o evaluación iluminativa, corresponde a la tercera generación y aparece en 1972, establecida por Parlett y Hamiliton (2007). Se caracteriza por introducir el término de juicio en el proceso de evaluación. El evaluador toma el papel de juez y su función ya no radica únicamente en obtener información sino, igualmente, en procesar sistemáticamente para la toma de decisiones. Ante ello, Muñoz (2007: 161) asevera: "El juicio del evaluador es el que determina la naturaleza de la evaluación".

Por otro lado, Stenhouse, MacDonald y Elliot proponen la evaluación democrática. Macdonald (1977) clasifica la evaluación en burocrática, autocrática y democrática. Cabe aclarar que, a pesar de su nominación democrática, no se fundamenta en principios democráticos dado que la evaluación está en quién la ejecuta, sin considerar otros factores. El evaluador toma un papel de intermediario de la información, mientras que en la evaluación burocrática es un ayudante contratado, y en la autocrática, un asesor experto. Se le denominó democrática porque los datos obtenidos permiten documentar la acción humana de forma individual. Lo esencial de este tipo de evaluación radica en saber en qué y cómo utilizar la información.

En el trascurso de la cuarta generación surge la evaluación de negociación, conocida como constructivista; comprende los resultados de la evaluación y construcciones representativas de los actores. Este tipo de evaluación mantiene la disposición hacia la toma de decisiones y, a la vez, se logra mediante la negociación. El evaluador deja a un lado el papel de juez para ser un investigador, permitiéndole entender el proceso de aprendizaje, incluso, saber valorarlo. Otra particularidad de esta generación es que la evaluación debe ser continua y oportuna a fin de ejercer una toma de decisiones de forma más acertada. La autoevaluación y la metaevaluación empiezan a figurar. Desde otros ángulos, Gómez (1993) reorganiza la evaluación respondiente; Stake (1967) la evaluación iluminativa; Parlett y Hamiliton (1972), la evaluación democrática; y Stenhouse, MacDonald y Elliot (1977) los submodelos de la evaluación de negociación.

La quinta generación se distingue por el paradigma sistemático de evaluación institucional o para la calidad, definido por Muñoz (1992: 184) como:

[...] un marco conceptual para la indagación de problemas en el área de la evaluación, buscando respuestas originales y creativas basadas en la teoría general de los sistemas y la teoría organizacional que les es propia, dichas respuestas, los procesos, los procedimientos y las proposiciones concretas, producto de la indagación hecha, no serán validables en y por sí mismas, sino a través de su acción.

Diólopos sobre Educación año 11 | número 21 | julio-diciembre 2020 | ISSN 2007-2171 
A la evaluación institucional se le adjudica un carácter ecléctico puesto que se basa en algunos principios de anteriores paradigmas, por ejemplo la evaluación democrática, al considerar como nodal a quien evalúa. Partiendo de ello, se aprecian tres características esenciales de la evaluación institucional: en primer lugar, a la evaluación se le concibe como un proceso de investigación social; en segundo lugar, el proceso en la que se origina es sistemático y, por último, el concepto de institución converge con la concepción asumida.

Para efectos del estudio analítico, adicionalmente a los modelos de evaluación presentados por Muñoz (2007), se exponen otros más dado que permiten ampliar la visión acerca de la evaluación y facilitan la comprensión de su evolución: evaluación alternativa, de ejecución, auténtica, por competencias, y finalmente, la socioformativa.

La evaluación alternativa se basa en principios constructivistas y atiende con mayor énfasis las exigencias de la sociedad de la información, más que las de la sociedad del conocimiento. Se diferencia de la tradicional en que es más completa; esto se debe a dos razones: la primera, puesto que emplea diversos procedimientos y técnicas incorporados a las actividades diarias; y la segunda, porque al usar varios procedimientos y técnicas recopila evidencia más cercana a la realidad del proceso de aprendizaje. La diversidad de la instrumentación de evaluación y su metodología responden a la intención de adaptarse a diferentes situaciones y a los propios alumnos. Además, al obtener más información y de forma completa, genera mayor credibilidad en la evaluación.

Los rasgos que distinguen a esta evaluación son: favorece la evaluación formativa; considera los diferentes estilos de aprendizaje, capacidades y experiencias de los alumnos; hace énfasis en las fortalezas de los alumnos en lugar de sus debilidades o errores; propicia la reflexión del proceso de aprendizaje, por lo que, a su vez, da oportunidad a un perfeccionamiento en el proceso de enseñanza - aprendizaje; facilita la documentación del avance del alumno y del grupo; se lleva a cabo a través de actividades dinámicas participativas, compartidas y continuas; se genera la evaluación por distintos agentes (heteroevaluación, coevaluación y autoevaluación); $y$, por último, las pruebas permiten ensayos y aplicaciones.

Las técnicas de evaluación alternativas se clasifican en dos grupos: las del desempeño y de observación. La Secretaría de Educación Pública de México (2019: 1) facilita la clasificación de las técnicas en las carteras de registro de asistencia y evaluación para los docentes; además, hace énfasis en la ejecución de la evaluación formativa mediante el Acuerdo 11/02/2019: "Que la evaluación tiene una finalidad esencialmente formativa al constituirse en la fuente de información para el mejoramiento de la práctica educativa [...]". Las técnicas de evaluación de desempeño sirven para valorar el proceso y el producto final: pruebas escritas y orales, matrices de valoración, listas de cotejo, escalas de apreciación o estimativas, etc. Las técnicas de evaluación de observación son de carácter complementario y valoran más lo actitudinal; los instrumentos recurrentes son: guías de observación, registros anecdótico, diarios de clase o de trabajo, escalas de actitudes. 
En el marco del enfoque de evaluación alternativo subyacen otros dos modelos: evaluación de ejecución y la auténtica.

En la evaluación de ejecución, los alumnos deben demostrar, construir o desarrollar un producto que solucione un problema en condiciones definidas y estándares. Por otro lado, la evaluación auténtica se basa en la actuación de los alumnos en condiciones reales. Mateo y Martínez (2008) refieren a Khatri y Sweet (1996: 15), quienes sostienen que "los alumnos deben estructurar las tareas objeto de evaluación, aplicar una información previa, construir respuestas y explicar el proceso que los ha llevado a determinada respuesta". La evaluación auténtica es propuesta por Herman, Aschbar y Winters (1992) y retomada por Díaz (2010). Este tipo de evaluación se centra principalmente en el desempeño del alumno y valora los aprendizajes obtenidos de tareas activas y complejas en situaciones de la vida real, por lo que alude a una evaluación contextualizada y una enseñanza situada, es decir, se enfoca en el proceso y no en los resultados, como sucede en la evaluación sumativa. Es importante puntualizar la diferencia entre la evaluación formativa y la sumativa para discernir la relación de esta con la evaluación auténtica y con la evaluación basada en competencias. Scriven (1967) establece la distinción entre ambas y varios autores la aluden. Álvarez (2003: 27) manifiesta que la evaluación formativa consiste en una actividad cognitiva dado que se auxilia al alumno a "crecer y a desarrollarse intelectual, afectiva, moral y socialmente", por lo que la evaluación se coloca al servicio del aprendizaje. En lo que concierne a la evaluación sumativa, obedece más al cumplimiento de objetivos administrativos puesto que se centra en los resultados o para certificar el rendimiento académico del alumno al terminar de cursar una asignatura, como lo indica Moreno (2016).

La evaluación por competencias surge en la época en que se reemplaza la sociedad de la información con la del conocimiento. Mejía (2012: 32) menciona:

[...] es un paradigma curricular que prevé las necesidades educativas contemporáneas cimentadas en el desarrollo pleno de los sujetos, la multiculturalidad, el advenimiento masivo de la tecnología, los cambios climatológicos y sus repercusiones, la deformación sociocultural de los principios de jerarquía adquisitiva de bienes materiales y la carencia evidente de valores del bienestar común.

Se puede deducir que para efectos de la evaluación por competencias, tras varias aportaciones de teóricos especializados, el axioma de evaluación ha tenido conversiones conceptuales; la principal es que dejó de concebirse como objetivo de la misma y ahora se comprende como un proceso, por lo que coincide con la evaluación formativa. Varios autores han realizado distintas conceptualizaciones acerca de la evaluación por competencias, sin embargo, de todas las acepciones actuales, la más aceptada en Iberoamérica y los países europeos es la propuesta por Tobón(2013). Al respecto, este autor sostiene que la evaluación por competencias es "un proceso que busca el mejoramiento continuo con base en la identificación de logros y aspectos 
por mejorar en la actuación de las personas en la resolución de problemas del contexto" (2013: 24).

La evaluación por competencias se fundamenta en los lineamientos pedagógicos de la evaluación integral, que radica en valorar el desarrollo cognitivo, procedimental y actitudinal del alumno, los cuales constituyen los pilares de la Escuela Transformadora, así lo afirma lafranscesco (2005). De igual forma, este autor señala que se basa en los dos principios de la evaluación auténtica, ya que las competencias se evalúan en la acción y en situaciones reales. El primer principio se refiere a que no se debe emitir una evaluación con base en lo que los alumnos resuelven en una prueba escrita al final del curso, puesto que se limitaría a valorar los saberes declarativos y factuales; es necesario constatar sobre la marcha el desempeño del alumno ante circunstancias complejas y contextualizadas. El segundo principio de la evaluación auténtica es el planteamiento de situaciones cercanas a la vida cotidiana.

La evaluación requerida en la sociedad del conocimiento actual se conforma tanto de la metodología cuantitativa y cualitativa, pero no de manera análoga, sino que una es resultado de la otra; se cualifica para cuantificar. Dicho en otros términos, al exponer "La metodología para evaluar competencias descansa en la premisa de la valoración y estimación cuantitativa y cualitativa del progreso evidente de competencias diversas según criterios éticos y objetivos" (Mejía, 2012: 33). Por otra parte, Tobón (2013) aclara que en el nivel de preescolar de la educación básica, se emplea únicamente la categoría cualitativa.

En relación con aspectos didácticos, Díaz (2010) presenta acciones que facilitan la ejecución de la evaluación por competencias: definir la situación didáctica al alumno; diseñar actividades que conduzcan a un proceso evaluador abierto; que propicie la evaluación de competencias en sus diversas dimensiones, así como también la identificación del grado de los distintos componentes que conforman la competencia a evaluar, nominándolos indicadores de desempeño.

Además de conocer las vertientes metodológicas de la evaluación por competencias, tanto cualitativa como cuantitativa, y sus acciones didácticas, surge la cuestión acerca de cuáles son los elementos que la comprenden. La respuesta de Tobón (2013) ante esta interrogante es que son: el aprendizaje esperado a evaluar (SEP, 2018, 2011, 2006); las evidencias pertinentes o pruebas de ejecución (ensayos, mapas conceptuales, informes de prácticas, informes de ensayos, registros de roles, cartas, pruebas escritas, entre otros); la instrumentación para evaluarlas; y los niveles de desempeño y acciones de mejoramiento para los actores involucrados en la educación.

Otros aspectos relevantes en la evaluación por competencias son, en primer lugar: el manejo de diversos tipos de evaluación; el empleo de la evaluación por actores (heteroevaluación, coevaluación y autoevaluación) y, de igual forma, se requiere la evaluación según su temporalización (diagnóstica, procesual y final) y su funcionalidad (formativa). En segundo lugar destaca el papel de la retroalimentación en el proceso de evaluación, hecha con asertividad; y finalmen- 
te la metaevaluación, retomada de la evaluación de negocios, pero incluyendo las aportaciones de la evaluación socioformativa.

El enfoque socioformativo enriquece de forma sustancial el de competencias; un ejemplo de ello es su contribución a la evaluación. Tobón (2013), considerado también el precursor del enfoque socioformativo, reconsidera y complementa los cánones de la evaluación por competencias en ejes, para brindar mayor claridad y precisión en la metodología: formar y aplicar la competencia maestra de evaluación; establecer el propósito de la evaluación de las competencias; enfocarse en el logro de los aprendizajes esperados y en la realización de evidencias; plantear problemas del contexto en la evaluación; evaluar a través del portafolio; esbozar y utilizar rúbricas socioformativas; desarrollar la metacognición en la formación y la evaluación; diseñar y aplicar instrumentos de evaluación basados en problemas; y retroalimentar a los alumnos con asertividad.

De los preceptos de la evaluación por competencias expuestos por Tobón (2013), resulta necesario referir algunas aproximaciones conceptualuales a la metagonición para comprender su papel en el proceso de la evaluación socioformtiva. Arias y Aparicio (2020), Córdoba y Marroquín (2018), Hernández y Camargo (2017), Owo y lkwut (2017), Sonowal y Kalita (2017), Abdellah (2015), Osses y Jaramillo (2008), y Flores (2000) coinciden acerca de la significación que tiene la metacognición en el aprendizaje. El constructo de metacognición aparece en los años setenta con las colaboraciones de Flavell (1979), quien la concibe como el entendimiento acerca de los propios procesos del pensamiento, definición que a su vez coincide con la propuesta por Carretero (2001), al mencionar que la metacognición radica en que el sujeto conoce a través de las propias actividades cognitivas. Además, tanto Huertas, Vega y Galindo (2014) como Meza y Lazarte (2007) y Flavell (1979) atribuyen a la metacognición las funciones de conciencia y control de dichas actividades pero, a diferencia de Flavell, los primeros la conciben como un proceso de reflexión.

La metacognición es reformulada desde el enfoque socioformativo, aunque sigue conservando su carácter reflexivo. De acuerdo con lo propuesto por Tobón (2013: 3), la metacognición consiste en "demostrar mejoras concretas en la actuación con respecto a una determinada meta, con apoyo en la reflexión." Asimismo, la metacognición se realiza durante todo el proceso de desarrollo de la competencia y no únicamente en lo cognitivo, procedimental y condicional, como sostienen Sáiz, Carbonero y Flores (2010), Osses y Jaramillo (2008) y Bausela (2004). La metacognición socioformativa tiene como propósito mejorar la actuación del alumno, al tener conciencia acerca de sus procesos cognitivos, procedimentales y afectivos.

Otra de las diferencias consiste en que, en el enfoque socioformativo, la metacognición la puede efectuar una comunidad o equipo y no solamente de manera individual. Una tercera característica de la metacognición socioformativa, pero que coincide con la evaluación formativa, es que se concentra en obtener una meta para la resolución de problemas. Finalmente, la metacognición socioformativa se vincula con un proyecto ético de vida. 
Todas las características que se le atribuyen a la metacognición socioformativa son para aplicar la estrategia Metacognición Antes, Durante y al Final de la Actividad (MADFA), propuesta por Tobón (2013). Esta estrategia consiste en una serie de actividades a realizar en diferentes momentos durante el desarrollo de una competencia, en los que se ponen en juego los procesos de la actuación y autorregulación.

Mejía (2012: 32) sostiene que el enfoque tiene cimientos en contribuciones pedagógicas actuales:

[...] el currículo sociocognitivo complejo (Tobón, 2011), la quinta disciplina (Senge, 2000, 1994), el pensamiento complejo (Morin, 2000a), el paradigma sociocognitivo (Román y Díez, 2000, 1994; Román, 1999, 1998), la formación basada en competencias (Maldonado, 2001), la pedagogía conceptual (Zubiría, 1998) y el aprendizaje estratégico (Pozo y Monereo, 1999).

Al igual que el enfoque por competencias, el enfoque socioformativo se complementa con la teoría de educación emocional (Goleman, 2004), teoría de las inteligencias múltiples (Gardner, 1983), la enseñanza situada (Brown, Collins y Duguid, 1989) y el aprendizaje basado en problemas, sin dejar atrás las raíces del constructivismo social (Vygotsky, 1978). El enfoque socioformativo se suscribe en los planteamientos pedagógicos de la Educación para la Liberación y de la Dialogicidad, propuestos por Paulo Freire (1995), al argumentar que el diálogo constituye un obligatorio en el proceso de aprendizaje. Al respecto, Moreno (2016), Álvarez (2003) y Santos (1995) coinciden en que la evaluación debe comprenderse como un proceso de diálogo. Tocante a la metacognición, Osses y Jaramillo (2008) deducen que, igualmente, se origina a través de una evaluación dialogada.

Por otro lado, Álvarez (2003) indica que quien aprende debe conocer en dónde se encuentra, por lo que el docente debe informárselo en cada momento, y eso se logra mediante un constante diálogo entre ambos actores. La comunicación entre el docente y el alumno le otorga un papel más activo a este en la evaluación, y no solo para el mejoramiento del proceso de aprendizaje sino, de igual forma, en el proceso de enseñanza. Alcaraz, Fernández y Sola (2012) señalan que la inclusión del alumno en la evaluación facilita la mejora en las prácticas docentes con respecto a algunas particularidades, como tener libertad en la organización del trabajo, conexión entre la teoría y práctica, así como crear espacios en los que el alumno construya su pensamiento al confrontarlo con sus demás compañeros.

La evaluación socioformativa incluye la retroalimentación y la metaevaluación. Ambos términos son reformulados por Tobón: "La retroalimentación asertiva consiste en valorar las competencias de los alumnos con una comunicación clara, respetuosa y cordial en todo el proceso, desde el establecimiento de los propósitos de la evaluación y pasando por la ejecución del proceso hasta llegar a la retroalimentación final" (2013: 255). 
En lo que se refiere a la metaevaluación, el autor expone que es "proceso clave de la socioformación y consiste en hacer metacognición sobre la forma en la cual se está evaluando". Tobón (2017) afirma que la evaluación socioformativa reside en el proceso continuo, diagnóstico, de retroalimentación y de apoyo, tanto a organizaciones como a individuos cuyo propósito es lograr determinadas metas mediante el desarrollo del talento, para que la sociedad avance hacia la inclusión y el desarrollo sustentable.

La retroalimentación asertiva converge con lo indicado por Alcaraz (2016) en lo referente a la evaluación, al comprenderla como la devolución de datos útiles para saber qué influyó durante el proceso de aprendizaje con miras a su mejoramiento. Para ello, Alcaraz (2016) y Tobón (2013) retoman el portafolios como una de las principales herramientas en la evaluación por competencias. Alcaraz (2016) sugiere algunas condiciones para darle un uso al servicio del aprendizaje. La primera consiste en que el portafolios debe enfocarse en el análisis y reflexión de los procesos y contextos de aprendizaje; la segunda está en fomentar un clima de libertad para promover la comunicación y el aprendizaje auténtico; el tercer uso del portafolios es que el error se concibe como fuente de aprendizaje; mientras que el cuarto uso se basa en recoger la opinión del alumno y, por último, el portafolios en sí comprende un sistema de evaluación, por lo que su función se amolda más a la formativa que a la sumativa.

Como se puede advertir, los instrumentos per se no son mejores ni peores, sino que importa el empleo que se les dé en el proceso de evaluación y en el manejo de la información que se obtiene a través de ellos. Por ejemplo, si se emplean las rúbricas o listas de cotejo para evaluar únicamente resultados, y hasta el final del proceso de aprendizaje se está efectuando la evaluación sumativa y no formativa. Ante ello, Hernández-Mosqueda, Tobón y Guerrero (2017) proponen a la rúbrica socioformativa como un instrumento de evaluación. El instrumento se basa en la Taxonomía de la Socioformación, por lo que incluye tanto el aspecto cognitivo y el actitudinal. La rúbrica se conforma por niveles de dominio expuestos de forma ascendente en cuestión de complejidad: preformal, receptivo, resolutivo, autónomo, estratégico y proyectual. Cardona (2016: 428) menciona que "El uso de las rúbricas en el proceso de la evaluación de evidencias facilita la retroalimentación, permite señalar los retos progresivos a ser alcanzados por los alumnos y miden las acciones del alumnado sobre los aspectos de la actividad que serán evaluados".

Tobón (2016) establece los elementos clave y los ejes de la evaluación por competencia. Los elementos mínimos de esta evaluación sirven para el análisis equitativo del desempeño y estos son: metas claras y pertinentes, problemas prioritarios, trabajar en productos y procesos, instrumentos válidos y oportunos, retroalimentación asertiva, y las oportunidades de mejora y socialización de los productos y procesos.

Considerando los elementos para realizar la evaluación socioformativa, resulta básico saber en qué condiciones se propicia: gestión, organización, mejoramiento e implementación del 
trabajo colaborativo. Por otra parte, aunque se tengan las circunstancias para generar la evaluación socioformativa, antes de ejecutarla, se deben considerar los medios, recursos, claridad de los criterios y fines, activación de mejoras continuas y procesos colaborativos. Es importante acentuar que los criterios y fines se dan a conocer al inicio y deben estar sustentados en fuentes, así lo afirma Zabalza (1997).

\section{Consideraciones finales}

La revisión analítica de los modelos de evaluación brindó la oportunidad de conocer las ventajas y desventajas de los paradigmas de evaluación originados en las diferentes etapas que comprenden la evolución de la evaluación educativa, con base en el recorrido histórico de la literatura existente (Alcaraz, 2015; Mejía, 2012; Muñoz, 2007; Rivera de Mendoza y Piñero, 2006; Mora, 2004). Distinguir las cualidades y los inconvenientes de cada modelo de evaluación facilitó la adquisición de elementos teóricos necesarios para rectificar la repetición de aquellas debilidades que permanecen en el proceso de evaluación en la actualidad y, a su vez, replantear acciones de mejora en la evaluación partiendo del enfoque más acorde a las exigencias de la sociedad del conocimiento y del paradigma socioformativo.

En síntesis, el estudio analítico de los paradigmas de evaluación sirvió para comprender la evolución de la evaluación y, por lo tanto, se deduce que las aportaciones brindadas por los diferentes modelos analizados han influido positivamente en su evolución, dado que algunos se complementan al retomar aquellos preceptos que seguían respondiendo a las exigencias propias del proceso de enseñanza - aprendizaje; en otros casos se contraponen, y decretan nuevos principios que facilitan la mejora de la aplicación de la evaluación, como sucede en el caso de la evaluación sumativa y la formativa. Asimismo, se concluyó que el paradigma de evaluación que responde de forma más acorde y congruente a las demandas de la sociedad del conocimiento es el que promueve el enfoque socioformativo, debido a que incorpora varios axiomas indispensables para ello, la inclusividad y el desarrollo sostenible (Juárez-Hernández, Tobón, Salas-Razo, Jerónimo-Cano y Martínez-Valdés, 2019).

También se concluyó que la evaluación socioformativa es uno de los enfoques que más ha contribuido a la evolución de la evaluación, puesto que reformula elementos teóricos y metodológicos que proporcionan mayor claridad y precisión en su aplicación, tanto en el ámbito educativo como en el organizacional; algunas de sus contribuciones son: la retroalimentación asertiva, la metaevaluación y la metacognición. No obstante, es necesario reconocer que también retoma varios preceptos de los anteriores modelos, en concreto, de la evaluación alternativa y la auténtica.

Además, se advirtió que la evaluación socioformativa (Tobón, 2017) converge con la evaluación formativa (Moreno, 2016) en varios aspectos: el alumno se involucra en el proceso de evaluación; el docente y el alumno comparten metas; se genera la retroalimentación y la me- 
tacognición; se evidencia la progresión del aprendizaje dado que la mejora no se centra en los resultados sino en el proceso. Aunado a ello, se retomó el planteamiento de la metacognición desde el enfoque socioformativo a través de la prospección de la estrategia MADFA (Tobón, 2013), que consiste en poner en marcha acciones que propicien la metacognición durante el desarrollo de competencias y al considerarse todos los procesos que lo comprenden, cognitivos, procedimentales y afectivos.

El enfoque socioformativo, al reformular elementos teóricos y metodológicos, genera nuevas inquietudes para continuar la búsqueda de próximos hallazgos sobre el tema. Además, a través del análisis de la evolución de la evaluación y sus diversos paradigmas, fue posible apreciar que la retroalimentación también ha tenido su propia evolución. Resultaría importante y oportuno estudiarla más a fondo dado el papel sustancial que desempeña para ejercer una evaluación adecuada, particularmente en el marco del enfoque socioformativo.

\section{Referencias bibliográficas}

Abdellah, R. (2015). Metacognitive Awareness and its Relation to Academic Achievement and Teaching Performance of Pre-service Female Teachers. Ajman University in UAE. Procedia - Social and Behavioral Sciences 174, 560-567. DOI: 10.1016/j.sbspro.2015.01.707

Alcaraz, N._(2015). Aproximación histórica a la evaluación educativa: De la generación de la medición a la generación ecléctica. Revista lberoamericana de Evaluación Educativa, 8(1), 11-25.

Alcaraz, N. (2016). La evaluación a través de portafolios: ¿Una ocasión para el aprendizaje? Revista Iberoamericana de Evaluación Educativa, 9(1), 31-46. DOI: 10.15366/riee2016.9.1.002

Alcaraz, N.; M. Fernández Navas; M. Sola (2016). La voz del alumnado en los procesos de evaluación docente universitaria. Revista lberoamericana de Evaluación Educativa, 5(2), 27-39.

Álvarez, J. M. (2003) Aprender con la evaluación. Revista Contextos: Estudios de Humanidades y Ciencias Sociales, 10, 19-39.

Arias Barahona, R; A. S. Aparicio (2020). Conciencia metacognitiva en ingresantes universitarios de ingeniería, arquitectura y ciencias aeronáuticas. Propósitos y Representaciones, 8(1), e272. DOI: $10.20511 /$ pyr2020.v8n1.272

Bausela, E. (2004). Diseño de un sistema de categorías para evaluar el conocimiento metacognitivo (persona, tarea y estrategia) con relación a la expresión escrita. Revista Electrónica Diálogos Educativos ,8(4), 31-39.

Cardona, S.; J.Vélez; S. Tobón (2016). Contribución de la evaluación socioformativa al rendimiento académico en pregrado. EDUCAR, 52(2), 423-447. 
Carretero M. (2001). Metacognición y educación. Buenos Aires: Aique.

Córdoba, D.; H. M. Marroquín (2018). Mejoramiento del rendimiento académico con la aplicación de estrategias metacognitivas para el aprendizaje significativo. Revista UNIMAR, 36(1), 15-30. DOI: https://doi.org/10.31948/unimar.36-1.1

Díaz, F. (2010). Estrategias docentes para un aprendizaje significativo. Una interpretación constructivista. México: McGraw-Hill/Interamericana.

Flavell, J. H. (1979). Metacognition and Cognitive Monitoring: A New Area of Cognitive Developmental Inquiry. American Psychologist, 34(10), 906-911.

Flores, R. (2000). Autorregulación, metacognición y evaluación. Acción Pedagógica, 9(1), 4-11.

Hernández, A.; A. Camargo (2017). Autorregulación del aprendizaje en la educación superior en Iberoamérica: Una revisión sistemática. Revista Latinoamericana de Psicología, 49, 146-160. DOI: 10.1016/j.rlp.2017.01.001

Hernández-Mosqueda, J. S.; S. Tobón-Tobón; G. Guerrero-Rosas (2016). Hacia una evaluación integral del desempeño: las rúbricas socioformativas. Ra Ximhai, 12(6).

Huertas, A.; G. Vesga; M. Galindo (2014). Validación del instrumento Inventario de Habilidades Metacognitivas (MAl) con estudiantes colombianos. Praxis \& Saber. Revista de Investigación y Pedagogía, 5(10), 55-74. DOI: 10.19053/22160159.3022

lafrancesco, G. (2005). La evaluación integral y del aprendizaje. Fundamentos y estrategias. Bogotá: Magisterio.

Juárez-Hernández, L. G.; S. Tobón; G. Salas-Razo; A. E. Jerónimo-Cano; M. G. Martínez-Valdés (2019). Desarrollo sostenible: educación y sociedad. M+A. Revista Electrónica de Medioambiente, 20(1), 54-71.

Mateo, J. A.; F. Martínez (2008). La evaluación alternativa de los aprendizajes. Cuadernos de Docencia Universitaria. Barcelona: Octadero.

Mejía, O. (2012). De la evaluación tradicional a una nueva evaluación basada en competencias. Educare, 16(1), 27-46.

Meza, A.; C. Lazarte (2007). Manual de estrategias para el aprendizaje autónomo y eficaz. Lima: Universidad Ricardo Palma / Editorial Universitaria.

Mora, A. (2004). La evaluación educativa: Concepto, períodos y modelos. Revista Electrónica Actualidades Investigativas en Educación, 4(2).

Moreno, T. (2016). Evaluación del aprendizaje y para el aprendizaje. México: Universidad Autónoma Metropolitana.

Muñoz, G. (1992). Paradigma sistémico de evaluación institucional. Maracay: Centro de Investigación en Educación de Calidad (Ceineduca).

Muñoz, G. (2007). Un nuevo paradigma: La quinta generación de evaluación. Laurus, 13(23), 158-198. 
Osses, S.; S. Jaramillo (2008). Metacognición: Un camino para aprender a aprender. Estudios Pedagógicos, 34(1), 187-197. DOI: $10.4067 /$ S0718-07052008000100011

Owo, W.; F. Ikwut (2015). Relationship between Metacognition, Attitude and Academic Achievement of Secondary School Chemistry Students in Port Harcourt, Rivers State. Journal of Research \& Method in Education, 5(6), 6-12. DOI: 10.9790/7388-05630612

Rivera de Mendoza, M.; M. Piñero Martín (2006). La generación emergente en la evaluación de los aprendizajes: Concepciones y modelos. Laurus, 12(22), 26-48.

Sáiz, M.; V. Flores; J. Román (2010). Metacognición y competencia de "aprender a aprender" en la educación infantil: Una propuesta para facilitar la inclusión. Revista Electrónica Interuniversitaria de Formación del Profesorado, 13(4), 123-130.

Santos, M. A. (1993). La evaluación, un proceso de diálogo, comprensión y mejora. España: Aljibe. Secretaría de Educación Pública (2019). Acuerdo número 11/03/19. https://bit.ly/2UvXUKC

Secretaría de Educación Pública (2018). Aprendizajes Clave. Español. Plan y programas de estudio, orientaciones didácticas y sugerencias de evaluación. México: SEP.

Secretaría de Educación Pública (2011). Programas de Estudio (2011) Español. Secundaria. Guía para el maestro. Educación Básica. México: SEP.

Secretaría de Educación Pública (2006). Programas de Estudio (2006) Español. Secundaria. Guía para el maestro. Educación Básica. México: SEP.

Sonowal, M.; M. Alita (2017). Metacognitive Awareness and Academic Achievement of Higher Secondary Level Science Stream Students of Dibrugarh Distric, Assam. International Education \& Research Journal, 3(6), 76-79.

Tobón, S. (2017). Evaluación socioformativa. Florida: Kresearch.

Tobón, S. (2013). Evaluación de las competencias en la educación básica. México: Santillana.

Tobón, S. (2013). Formación integral y competencias: Pensamiento complejo, currículo, didáctica y evaluación. Bógota: ECOE.

Tobón, S. (2013). Proceso metacognitivo y estrategia MADFA. México: CIFE

Tobón, S.; J. Pimienta; J. A. García (2010). Secuencias didácticas: Aprendizaje y evaluación de competencias. México: Pearson.

Zabalza, M. A. (1997). Diseño y desarrollo curricular. Madrid: Narcea.

Diálo oos 